\title{
Forecasting Return Volatility of the CSI 300 Index Using the Stochastic Volatility Model with Continuous Volatility and Jumps
}

\author{
Xu Gong, Zhifang He, Pu Li, and Ning Zhu \\ School of Business, Central South University, Changsha, Hunan 410083, China \\ Correspondence should be addressed to Ning Zhu; zhuning@csu.edu.cn
}

Received 13 April 2014; Accepted 12 June 2014; Published 9 July 2014

Academic Editor: Chuangxia Huang

Copyright @ $2014 \mathrm{Xu}$ Gong et al. This is an open access article distributed under the Creative Commons Attribution License, which permits unrestricted use, distribution, and reproduction in any medium, provided the original work is properly cited.

\begin{abstract}
The logarithmic realized volatility is divided into the logarithmic continuous sample path variation and the logarithmic discontinuous jump variation on the basis of the SV-RV model in this paper, which constructs the stochastic volatility model with continuous volatility (SV-CJ model). Then, we use high-frequency transaction data for five minutes of the CSI 300 stock index as the study sample, which, respectively, make parameter estimation on the SV, SV-RV, and SV-CJ model. We also comparatively analyze these three models' prediction accuracy by using the loss functions and SPA test. The results indicate that the prior logarithmic realized volatility and the logarithmic continuous sample path variation can be used to predict the future return volatility in China's stock market, while the logarithmic discontinuous jump variation is poor at its prediction accuracy. Besides, the SV-CJ model has an obvious advantage over the SV and SV-RV model as to the prediction accuracy of the return volatility, and it is more suitable for the research concerning the problems of financial practice such as the financial risk management.
\end{abstract}

\section{Introduction}

Recent papers (such as Corsi [1], Wen and Yang [2], Liu et al. [3], Andersen et al. [4, 5], Dai et al. [6], Wen et al. [7], Bollerslev et al. [8, 9], and Liu et al. [10-12]) have showed that the financial risk management, financial asset pricing, and financial derivatives pricing play more and more important roles in the analysis of the problems in financial practices. What is more, the research on the asset volatility of the financial market is the basis of the analysis of the problems in financial practices like the financial risk management, financial asset pricing, and financial derivatives pricing. Therefore, the measurement and forecast of financial asset volatility have become hot topics.

In order to accurately measure and predict the financial asset volatility, Engle [13] proposed an ARCH model according to "clustering" and "long-memory" features of the return volatility; Bollerslev [14], on the basis of the ARCH model, established a GARCH model. Taylor [15] first proposed a stochastic volatility (SV) model. Then, many scholars study the SV model and comparatively analyze the measurement and forecast accuracy for financial asset volatility between the GARCH model and SV model. Among them, there are many literatures about the comparison of SV model and GARCH model on asset volatility measurement and sample fitting ability. Danielsson [16] studied the S\&P 500 index of American stock markets, and he found that the fitting ability of SV model for the S\&P 500 index volatility is stronger than the ARCH (5), GARCH $(1,2)$, IGARCH, and EGARCH $(2,1)$ model. Wang et al. [17], the empirical study on the application of China's stock market data, found that the SV model can better describe the heteroscedasticity in the return of stock market and the serial correlation of volatility than GARCH model. Kim et al. [18] also found that the SV model have a better sample fitting ability for the financial asset volatility than the GARCH model. In addition, there exist some literatures about the prediction accuracy of the future financial asset volatility as to the comparative study on SV model and GARCH model. Yu [19], the comparative study on the SV model and GARCH model, found that the SV model showed much better out-of-sample forecasting performance 
than the ARCH and GARCH $(1,1)$ and $\operatorname{GARCH}(3,2)$ model in New Zealand stock market. Sadorsky [20] (in the US stock market), Pederzoli [21] (in the UK stock market), and Wei [22] (in the crude oil futures market) came to a similar conclusion with $\mathrm{Yu}$ [19], that is to say, the SV model's prediction accuracy is stronger than the GARCH model's.

Although the SV model has good forecasting performance for the future return volatility, higher accuracy is more favorable to the analysis of practical financial problems such as financial risk measuring, financial asset pricing, and financial derivatives pricing. In order to improve the prediction and measurement accuracy of the model, Koopman et al. [23] introduced realized volatility (RV; Andersen and Bollerslev [24]) as an exogenous variable into the volatility equation of SV model so as to construct the SV-RV model. After applying the S\&P 100 index in American stock markets, Koopman et al. found the measuring and forecasting accuracy for return volatility of SV-RV model is stronger than the SV model. Then, Geweke et al. [25] found that the SV-RV model has a good ability to predict financial asset volatility. Jacquier and Miller [26] also found that the realized volatility (RV) contained certain prediction information for the future volatility, and the SV-RV model's prediction ability is superior to the SV model. However, in the real financial market, because of the impact of the abnormal information and the existence of the irrational investors, the financial asset volatility is not only a continuous process but also there are some jumps in it. Therefore, while we study the SV-RV model, it is more reasonable to divide the RV into the continuous sample path variation $(\mathrm{C})$ and the discontinuous jump variation $(\mathrm{J})$ and add the two factors into the volatility equation of SV model. Hence, after learning from Barndorff-Nielsen and Shephard $[27,28]$ and Andersen et al. [5], on the basis of the SV-RV model, we divide the RV into C and J and establish the SV-CJ model so as to further improve the model's ability to measure and forecast financial asset volatility. Then, we use the highfrequency data for five minutes of CSI 300 index in China's stock market as the research sample to make parameter estimation on the SV, SV-RV, and SV-CJ model, respectively, and use the loss functions and SPA test proposed by Hansen [29] to comparatively analyze forecasting performance for the future return volatility of the three models. By this way, we look for the best model for forecasting financial asset volatility.

The remainder of this paper is organized as follows. In Section 2, we discuss three volatility models, the SV, SV$\mathrm{RV}$, and SV-CJ model. In Section 3, we introduce estimation and evaluation method of the models. In Section 4, the estimating and forecasting results are presented. Section 5 is the conclusion of this paper.

\section{Volatility Models}

2.1. The $S V$ and $S V-R V$ Model. In the existing literature, there exist many forms about the SV model; one of the common forms can be expressed as follows:

$$
\begin{gathered}
y_{t}=\varepsilon_{t} e^{h_{t} / 2}, \\
h_{t}=\alpha+\beta h_{t-1}+\eta_{t},
\end{gathered}
$$

where $y_{t}$ is a return. $\left\{\varepsilon_{t}\right\}$ and $\left\{\eta_{t}\right\}$ are mutual dependent. In this paper, we suppose $\varepsilon_{t} \sim$ i.i.N $(0,1), \eta_{t} \sim$ i.i. $N\left(0, \sigma_{\eta}^{2}\right)$, and $\sigma_{\eta}^{2}$ is unknown. $\alpha$ and $\beta$ are constant. $\beta$, as continuous parameter, reflects the impact of the prior volatility on the current volatility, and when $|\beta|<1$, it stands for covariance stationary of the SV model. $h_{t}$ is the logarithm of return volatility; supposing $h_{0} \sim N\left(\alpha, \sigma_{\varepsilon}^{2}\right)$, we can conclude that for given $h_{t-1}, \alpha, \beta, h_{t}$ obeys normal distribution with mean $\alpha+\beta h_{t-1}$ and variance $\sigma_{\varepsilon}^{2}$; that, is $h_{t} \mid h_{t-1}, \alpha, \beta \sim N(\alpha+$ $\left.\beta h_{t-1}, \sigma_{\varepsilon}^{2}\right), t=1,2, \ldots, T$.

To enhance the model's accuracy for volatility measurement and prediction, according to Koopman et al. [23], we add $\ln \left(\mathrm{RV}_{t-1}\right)$ as an exogenous variable to the volatility equation of SV model; we establish the SV-RV model

$$
\begin{gathered}
y_{t}=\varepsilon_{t} e^{h_{t} / 2}, \\
h_{t}=\alpha+\beta h_{t-1}+\gamma \ln \left(\mathrm{RV}_{t-1}\right)+\eta_{t},
\end{gathered}
$$

where $\mathrm{RV}_{t-1}$ is a realized volatility at time $t-1$; the volatility used in this paper is identical to that in Martens [30] and Koopman et al. [23]; taking the overnight return variance into consideration, $\mathrm{RV}_{t-1}$ can be expressed as follows:

$$
\mathrm{RV}_{t}=\sum_{i=1}^{N} r_{t, j}^{2}+r_{t, n}^{2}=\sum_{j=1}^{M} r_{t, j}^{2}, \quad M=N+1,
$$

where $r_{t, 1}$ and $r_{t, n}$ stand for the overnight return, $r_{t, 1}=r_{t, n}=$ $100\left(\ln P_{t, o}-\ln P_{t-1, c}\right), P_{t, o}$ represents the open price at time $t$, and $P_{t-1, c}$ denotes the closing price of phase $t-1 ; r_{t, 2}$ is the 1st return after the opening of time $t, r_{t, 2}=100\left(\ln P_{t, 1}-\ln P_{t, o}\right)$; $P_{t, 1}$ is the 1st close price after the opening of time $t ; r_{t, 3}$ shows the 2nd return after the opening of time $t, r_{t, 3}=100\left(\ln P_{t, 2}-\right.$ $\left.\ln P_{t, 1}\right) ; \ldots ; r_{t, M}$ is the $(M-1)$ th return after the opening of time $t$, and $r_{t, M}=100\left(\ln P_{t, M-1}-\ln P_{t, M-2}\right)$.

2.2. The SV-CJ Model. In the real financial market, since the impact of information and irrational behavior of investors, the volatility of return on asset is no longer continuous, while there are some jumps. Andersen et al. [31] have shown that the separation of the realized volatility into the continuous sample path variation and the discontinuous jump variation will enhance the accuracy to predict future volatility. Therefore, in order to further improve the accuracy to predict the future volatility of the model, we transform the logarithmic realized volatility $\ln \left(\mathrm{RV}_{t}\right)$ of model (2) to the logarithmic continuous sample path variation $\ln \left(C_{t}\right)$ and the discontinuous jump variation $\ln \left(J_{t}+1\right)$.

When the realized volatility is divided into the continuous sample path variation and the discontinuous jump variation, we need to understand several important concepts. Please assume that return on assets is a continuous process; when we use the quadratic variation (quadratic variation, QV) to describe the total variation of the return volatility on financial assets and the integrated variation (IV) to depict the continuous part of the total variation, we can conclude that the difference between quadratic variation and integrated variation is the jump variation. In fact, the observed 
data are discrete, when they are used by the scholars to estimate the quadratic variation and integrated variation; the realized volatility and realized bipower variation (RBV) can be renamed. Barndorff-Nielsen and Shephard [27, 28] used the quadratic variation theory to separate realized volatility into the continuous sample path variation and the discontinuous jump variation. A mathematical description of this decomposition approach is given as follows.

Let $p_{t}=\ln \left(P_{t}\right)$ denote a logarithmic financial asset price at time $t$. The continuous-time jump diffusion process traditionally used in financial asset pricing is conveniently expressed in stochastic differential equation form as

$$
d p_{t}=\mu_{t} d t+\sigma_{t} d W_{t}+\kappa_{t} d q_{t}, \quad 0 \leq t \leq T
$$

where $\mu_{t}$ is a continuous and locally bounded variation process. $\sigma_{t}$ is a strictly positive stochastic volatility process with a sample path that is right continuous and has welldefined left limits (allowing for occasional jumps in volatility). $W_{t}$ is a standard Brownian motion. $\kappa_{t}$ refers to the size of the corresponding discrete jumps in the logarithmic price process. $q_{t}$ represents Poisson counting process of $\lambda_{t}$, and $\lambda_{t}$ is a time-varying intensity variable, so $P\left(d q_{t}=1\right)=\lambda_{t} d t$.

For discrete prices from a continuous time process, the logarithmic return volatility at time $t$ is a compound volatility including jump volatility rather than an unbiased estimator of integrated volatility. The log return rate from $t-1$ to $t$ is quadratic variation:

$$
\mathrm{QV}_{t}=[r, r]_{t}=\int_{t-1}^{t} \sigma_{s}^{2} d s+\sum_{t-1<s \leq t} \kappa_{s}^{2},
$$

where $\int_{t-1}^{t} \sigma_{s}^{2} d s<\infty$ is called an integrated variation, representing the continuously altering part of variation of the return rate. Besides, $\sum_{t-1<s \leq t} \kappa_{s}^{2}$ is called a jump volatility, representing the cumulative amount of jump variation of return rates in $[t-1, t]$.

Andersen and Bollerslev [24] argued that for quadratic variation, which cannot be observed directly, $\mathrm{RV}_{t}$ is a consistent estimator of $\mathrm{QV}_{t}$, when using the discrete data to calculate quadratic variation with $M$ converging to infinite

$$
\mathrm{RV}_{t} \stackrel{M \rightarrow \infty}{\longrightarrow} \mathrm{QV}_{t}=\int_{\mathrm{t}-1}^{\mathrm{t}} \sigma_{\mathrm{s}}^{2} \mathrm{ds}+\sum_{\mathrm{t}-1<\mathrm{s} \leq \mathrm{t}} \kappa_{\mathrm{s}}^{2}
$$

Besides, integrated volatility can be estimated by realized bipower variation (Barndorff-Nielsen and Shephard [27, 28]), which is consistent estimator of the continuous sample path variation, with $M$ converging to infinite

$$
\mathrm{RBV}_{t}=z_{1}^{-2} \frac{M}{M-2} \sum_{j-3}^{M}\left|r_{t, j-2}\right|\left|r_{t, j}\right|,
$$

where $z_{1}=E\left(Z_{t}\right)=\sqrt{\pi / 2} ; Z_{t}$ is a random variable subjecting to standardized normal distribution. $M /(M-2)$ is the correction of the sample size. According to BarndorffNielsen and Shephard [27, 28], when $M \rightarrow \infty$, the difference between $\mathrm{RV}_{t}$ and $\mathrm{RBV}_{t}$ is consistent estimator of the discontinuous jump variation:

$$
\mathrm{RV}_{t}-\mathrm{RBV}_{t} \stackrel{M \rightarrow \infty}{\longrightarrow} J_{t} .
$$

However, the discontinuous jump variation mentioned above cannot guarantee the result to be nonnegative for finite-sized sample. Therefore, to ensure the nonnegativity of the discontinuous jump variation, this paper handles the discontinuous jump variation $J_{t}$ as follows:

$$
J_{t}=\max \left[\mathrm{RV}_{t}-\mathrm{RBV}_{t}, 0\right] .
$$

In the computational process of the discontinuous jump variation, the arithmetic error varies with the frequency of sample selection. To improve the accuracy of the discontinuous jump variation, we use some estimator to test the level of significance of it. This paper applies $Z_{t}$ (BarndorffNielsen and Shephard [27, 28]; Huang et al. [32]) to identify the factors of discontinuous jump variation:

$$
\begin{aligned}
& Z_{t}=\frac{\left(\mathrm{RV}_{t}-\mathrm{RBV}_{t}\right) \mathrm{RV}_{t}^{-1}}{\sqrt{\left(\mu_{1}^{-4}+2 \mu_{1}^{-2}-5\right)(1 / M) \max \left(1, \mathrm{RTQ}_{t} / \mathrm{RBV}_{t}^{2}\right)}} \\
& \longrightarrow N(0,1),
\end{aligned}
$$

where $\mu_{1}=\sqrt{2 / \pi}$ and $\mathrm{RTQ}_{t}$ is realized tri-power quarticity:

$$
\begin{gathered}
\mathrm{RTQ}_{t}=M \mu_{4 / 3}^{-3}\left(\frac{M}{M-4}\right) \sum_{j=4}^{M}\left|r_{t, j-4}\right|^{4 / 3}\left|r_{t, j-2}\right|^{4 / 3}\left|r_{t, j}\right|^{4 / 3} \\
\mu_{4 / 3}=E\left(\left|Z_{T}\right|^{4 / 3}\right)=2 \frac{2}{3} \Gamma\left(\frac{7}{6}\right) \Gamma\left(\frac{1}{2}\right)^{-1} .
\end{gathered}
$$

Because of the remarkable relativity between the result of RBV and the possibility of high-frequency sample, with the increase of sampling frequency, the estimator of RBV can not be converged to the integrated volatility under the impact of the market microstructure. Therefore, it is biased to exploit $\mathrm{RBV}$ as the estimator of the robust test of discontinuous jump variation and thus this paper chooses the new estimator MedRV $_{t}$ (Andersen et al. [5]), which is

$$
\begin{aligned}
\operatorname{MedRV}_{t}= & \frac{\pi}{6-4 \sqrt{3}+\pi}\left(\frac{M}{M-2}\right) \\
& \times \sum_{i=2}^{M-1} \operatorname{Med}\left(\left|r_{t, i-1}\right|\left|r_{t, i}\right|\left|r_{t, i+1}\right|\right)^{2} .
\end{aligned}
$$

Correspondingly, $\mathrm{RTQ}_{1, t}$ of statistics $Z_{t}$ in (10) is also replaced by MedRTQ ${ }_{t}$, which is proposed by Andersen et al. [6] and can be defined by

$$
\begin{aligned}
\text { MedRTQ }_{t}= & \frac{3 \pi M}{9 \pi+72+-52 \sqrt{3}}\left(\frac{M}{M-2}\right) \\
& \times \sum_{i=2}^{M-1} \operatorname{Med}\left(\left|r_{t, i-1}\right|,\left|r_{t, i}\right|,\left|r_{t, i+1}\right|\right)^{4} .
\end{aligned}
$$


In (7), we replace $\mathrm{RBV}_{t}, \mathrm{MedRTQ}_{t}$ by MedRV after the calculation of statistic $Z_{t}$, under the significance level of $1-\alpha$; we can get the estimator of the discontinuous jump variation:

$$
J_{t}=I\left(Z_{t}>\phi_{\alpha}\right)\left(\mathrm{RV}_{t}-\mathrm{MedRV}_{t}\right)
$$

Correspondingly, the estimator of the continuous sample path variation is

$$
C_{t}=I\left(Z_{t} \leq \phi_{\alpha}\right) \mathrm{RV}_{t}+I\left(Z_{t}>\phi_{\alpha}\right) \mathrm{MedRV}_{t}
$$

In the actual computational process, we need to select the sound confidence level $\alpha$. In this paper, based on previous researches (such as Andersen et al. [4, 31]; Huang and Tauchen [32], Huang et al. [33]), confidence level $\alpha$ is set at 0.99 . In addition, through the above inspection of statistic $Z_{t}$ and based on the quadratic variation theory, we can get the logarithmic volatility estimator of the continuous sample path variation $C_{t}$ and the discontinuous jump variation $J_{t}$.

According to the decomposition method of realized volatility, we decompose $\mathrm{RV}_{t-1}$ into the continuous sample path variation $C_{t-1}$ and the discontinuous jump variation $J_{t-1}$. Referenced to the research of Andersen et al. [31], we can, respectively, transform $C_{t-1}$ and $J_{t-1}$ to logarithmic form $\ln \left(C_{t-1}\right)$ and $\ln \left(J_{t-1}+1\right)$. Then, adding $\ln \left(C_{t-1}\right)$ and $\ln \left(J_{t-1}+1\right)$ as exogenous variables followed the way of SV-RV model into the volatility equation of SV model; we can get the SV-CJ model:

$$
\begin{gathered}
y_{t}=\varepsilon_{\mathrm{t}} e^{h_{t} / 2} \\
h_{t}=\alpha+\beta h_{t-1}+\lambda \ln \left(C_{t-1}\right)+\theta \ln \left(J_{t-1}+1\right)+\eta_{t} .
\end{gathered}
$$

\section{Estimation and Evaluation Method}

3.1. Estimation Method. In the SV model, using maximum likelihood estimation method for parameter estimation is difficult, so there are many alternative methods produced, such as the moment method (Taylor, [15]), the pseudomaximum likelihood method (Ruiz [34]), the Markov Chain Monte Carlo method (MCMC; Jacquier et al. [35]), the generalized moment method (Andersen and Sørensen [36]), and the nonlinear filtering maximum likelihood method (Watanabe [37]). However, Jacquier [35], Kim et al. [18], and Durbin and Koopman [38] show that the MCMC method estimates in estimation performance are the best. Bauwens and Lubrano [39] pointed out that when using the MCMC method to estimate model parameters, using Gibbs sampling is better than importance sampling and Metropolis Hastings algorithm. The MCMC with the Gibbs sampling method can make full use of the advantages of computer simulation technology and get a large number of state samples. It uses elementary method to estimate model parameters and avoids the complicated calculation in E-M algorithm, so it improves the success rate of the estimate. Therefore, in this paper, using the MCMC method to estimate the parameters of SV, SV$\mathrm{RV}$, and SV-CJ model, the sampling method is the Gibbs sampling; the used software is the Open BUGS.

\subsection{Evaluation Method}

3.2.1. DIC Criterion. The SV, SV-RV, and SV-CJ model have many unknown variables, and the unknown variables are not independent of each other, and we are not able to determine the number of independent parameters in advance. In order to make a comparison among the goodness of SV, SV$\mathrm{RV}$, and SV-CJ model, we select the deviance information criterion (DIC) mentioned by Spiegelhalter et al. [40] to be the criterion of model evaluation. Mathematics form of DIC can be expressed as follows.

Dempster [41] considered that posterior distribution inspecting the classical deviation can employ Bayesian model, that is:

$$
D(\omega)=-2 \ln (L(y \mid \omega))+2 \ln (g(y)),
$$

where $\omega$ can represent $\alpha, \beta, \gamma, \lambda, \theta$, and logarithmic potential volatility sequences $\left\{\omega_{t}\right\}$. y refers to a list of data distribution, $y=\left(y_{1}, \ldots, y_{n}\right) . L(y \mid \omega)$ means likelihood function. $\ln (g(y))$ is the standardized form of independent data function. Bauwens and Lubrano [39] based on (17) develop into an important model selection criterion of DIC. DIC includes two parts, the specific expression as follows:

$$
\mathrm{DIC}=\bar{D}+p_{D}
$$

In this formula, the first part $\bar{D}$ is minus twice the posterior mean log-likelihood; a natural choice for a suitable model is one that minimizes the DIC. The posterior mean deviation is defined as a parameter. Consider

$$
\bar{D}=E_{\omega \mid y}[D(\omega)]=E_{\omega \mid y}[-2 \ln (L(y \omega))] .
$$

The second part $p_{D}$ is defined as the difference between the posterior mean of the deviance and the deviance evaluated at the posterior mean or mode of the relevant parameters. Consider

$$
\begin{aligned}
p_{D}=\bar{D}-D(\bar{\omega}) & =E_{\omega \mid y}[D(\omega)]-D\left(E_{\omega \mid y}(\omega)\right) \\
& =E_{\omega \mid y}[-2 \ln (L(y \mid \omega))]+2 \ln (L(y \bar{\omega})) .
\end{aligned}
$$

$\bar{\omega}$ is the posterior mean of $\omega . L(y \mid \bar{\omega})$ is the known parameters and logarithmic potential fluctuations of the likelihood function of average cases.

AIC is similar to AIC or BIC; the smaller the value of DIC, the better the model. But if we consider the model for the complexity of the data fitting ability, DIC has better comparative superiority and inferiority complex model than the AIC and BIC. In this paper, the SV, SV-RV, and SV-CJ model are complicated, so using DIC is more suitable.

3.2.2. Loss Functions. In this paper, we use the loss functions and SPA test with the "Bootstrap" to analyze the predictive accuracy of SV, SV-RV, and SV-CJ model both in sample and out of sample. According to Bollerslev et al. [42] and Hansen and Lunde [43], we choose six common loss functions. They are the mean absolute error (MAE, denoted by $L_{1}$ ), 
the heteroskedastic adjusted mean absolute error (HMAE, denoted by $L_{2}$ ), the mean squared error (MSE, denoted by $L_{3}$ ), the heteroskedastic adjusted mean squared error (HMSE, denoted by $L_{4}$ ), QLIKE (denoted by $L_{5}$ ), and $R^{2}$ LOG (We can refer the details of loss functions QLIKE and R2LOG to Bollerslev et al. [43] and Hansen and Lunde [43]) (denoted by $L_{6}$ ). If the values of the six functions are smaller, that means the corresponding predictive accuracy of volatility models are stronger. The computation expression of MAE, HMAE, MSE, HMSE, QLIKE, and $R^{2} L O G$ is as formulas (21). Because the volatility in the stock market cannot be observed, scholars (such as Koopman et al. [23]; Corsi [1]) often use $\mathrm{RV}_{t}$ to replace the real volatility at time $t$; therefore, we also use $\mathrm{RV}_{t}$ to replace the real volatility in stock market. Consider

$$
\begin{aligned}
& L_{1}: \mathrm{MAE}=\frac{1}{n} \sum_{t=1}^{n}\left|\sigma_{t}^{2}-\widehat{\sigma}_{t}^{2}\right| \\
& L_{2}: \mathrm{HMAE}=\frac{1}{n} \sum_{t=1}^{n}\left|\frac{\sigma_{t}^{2}-\widehat{\sigma}_{t}^{2}}{\sigma_{t}^{2}}\right| \\
& L_{3}: \mathrm{MSE}=\frac{1}{n} \sum_{t=1}^{n}\left(\sigma_{t}^{2}-\widehat{\sigma}_{t}^{2}\right)^{2} \\
& L_{4}: \mathrm{HMSE}=\frac{1}{n} \sum_{t=1}^{n}\left[\frac{\sigma_{t}^{2}-\widehat{\sigma}_{t}^{2}}{\sigma_{t}^{2}}\right]^{2} \\
& L_{5}: \mathrm{QLIKE}=\frac{1}{n} \sum_{t=1}^{n}\left[\ln \left(\widehat{\sigma}_{t}^{2}\right)+\frac{\sigma_{t}^{2}}{\widehat{\sigma}_{t}^{2}}\right] \\
& L_{6}: R^{2} \mathrm{LOG}=\frac{1}{n} \sum_{t=1}^{n}\left[\ln \left(\frac{\sigma_{t}^{2}}{\widehat{\sigma}_{t}^{2}}\right)\right]^{2},
\end{aligned}
$$

where $n$ is the number of predicted samples. $\sigma_{t}^{2}$ is real volatility, that is, $\mathrm{RV}_{t} . \widehat{\sigma}_{t}^{2}$ represents the prediction value of volatility obtained by the SV, SV-RV, or SV-CJ model.

3.2.3. SPA Test. On the basis of the loss functions, Hansen [29] proposed a superior predictive ability (SPA). Then, there are many researchers (such as Hansen and Lunde [43], Martin et al. [44], Wang and Wu [45], and Hung et al. [46]) who used this method to compare prediction accuracy of the models. Hansen [29] found that, due to SPA test with "Bootstrap," it has better model discriminated ability than RC test mentioned in White [47], and the SPA test has better robustness.

The SPA test can be simply described as follows. Firstly, we need to compare $J+1$ types of volatility models, denoted by $M_{k}, k=0,1, \ldots, J$. Every volatility model $M_{k}$ predict the future of volatility for a day as $\widehat{\sigma}_{k, t}^{2}, t=0,1, \ldots, n$. For each prediction, formula (21) can be calculated, as defined by the six loss functions, denoted by $L_{m, k, t}, m=1,2, \ldots, 6$. Using $M_{0}$ to be the inspection benchmark model of the SPA, $M_{k}(k=1,2, \ldots, J)$ is compared model. So we can calculate that $M_{0}$ are relative to compared model; the "relative loss function values" can be expressed as

$$
\begin{array}{r}
X_{k, t}=L_{m, 0, t}-L_{m, k, t} \\
m=1,2, \ldots, 6 ; k=1,2, \ldots, J ; t=1,2, \ldots, n .
\end{array}
$$

In order to determine whether there is a better prediction model than $M_{0}$ in $M_{k}(k=1,2, \ldots, J)$, we define a null hypothesis " $M_{0}$ is the best prediction model, compared with $M_{k}(k=1,2, \ldots, J)$." This null hypothesis can be expressed in mathematical expressions as follows:

$$
\max \lambda_{k}=E\left(X_{k, t}\right), \quad k=1,2, \ldots, J .
$$

Hansen [29] proved that the hypothesis test statistics are

$$
\begin{aligned}
& T=\max \frac{\sqrt{n} \bar{X}_{k, t}}{\widehat{w}_{k k}}, \quad k=1,2, \ldots, J, \\
& \text { in that } \bar{X}_{k, t}=n^{-1} \sum_{t=1}^{t=n} X_{k, t}, \quad \widehat{w}_{k k}=\operatorname{var}\left(\sqrt{n} \bar{X}_{k, t}\right) .
\end{aligned}
$$

In order to get formula (24) of the distribution of the $T$ statistic and $P$ value of Hansen [29] using "Bootstrap" to obtain the value is recommended. Firstly, we need to get a new sample $X_{k, t}$ of length $n$. To get a new sample, we need to randomly take a new subsample from the original collection $\left\{X_{k, t}\right\}$, and the length of the subsample from a obey averages $q$ geometric distribution of random numbers, and at the same time control the combination of these sub sample length required for $n$.

Repeating the Bootstrap process $B, B X_{k, t}$ of length $n$ can be obtained; that is, $X_{k, t}^{i}, \quad i=1,2, \ldots, B$. In this paper, $q=0.5$ and $B=5000$ times are used as the Bootstrap process control parameters. So each Bootstrap sample mean can be expressed as

$$
\bar{X}_{k}^{i}=n^{-1} \sum_{t=1}^{t=n} X_{k, t}^{i}, \quad i=1,2, \ldots, B .
$$

The estimator of $B$ Bootstrap samples mean variance can be expressed as

$$
\widehat{w}_{k k}=B^{-1} \sum_{i=1}^{i=B}\left(\bar{X}_{k}^{i}-\overline{\bar{X}}_{k}\right)^{2}, \quad \overline{\bar{X}}_{k}=B^{-1} \sum_{i=1}^{i=B} \bar{X}_{k}^{i} .
$$

Define $\bar{Z}_{k}^{i}$ as

$$
\begin{aligned}
& \bar{Z}_{k}^{i}=\left(\bar{X}_{k}^{i}-\overline{\bar{X}}_{k}\right) \times I\left(\overline{\bar{X}}_{k}>-A_{k}\right), \\
& \text { in that, } \quad A_{k}=\frac{1}{4} n^{-1 / 4} \widehat{w}_{k k} .
\end{aligned}
$$

$I\{\cdot\}$ is an indicator function; as the conditions of the $\{\cdot\}, I\{\cdot\}$ is 1 , otherwise is 0 . In the end, we can get the new $T^{i}$ statistical magnitude:

$$
T^{i}=\max \frac{\sqrt{n} \bar{Z}_{k}^{i}}{\widehat{w}_{k k}}, \quad i=1,2, \ldots, B .
$$


Hansen [29] showed that under the null hypothesis in (23), formula (28) $T^{i}$ statistical magnitude converges to formula (24), as defined by the $T$ statistic. Therefore, the $P$ values can be obtained directly from the type

$$
P=B^{-1} \sum_{i=1}^{i=B} I\left\{T^{i}>T\right\} .
$$

When comparing the quality prediction model and the test of SPA (the closer to 1 ), if the $P$ value is greater, we cannot refuse the null hypothesis of formula (27) any more. That is to say, compared with other models, the accuracy of the baseline model is much higher.

\section{Empirical Evidence}

4.1. Data and Summary Statistics. This paper uses the CSI 300 index in China's stock market to empirical evidence. The data derived from the WIND financial database. The time span of samples is April 20, 2007, to April 20, 2012, and is a total of 1199 days. In the calculation of realized volatility, the daily sample data extracting frequency greatly affects the result of the study. On the one hand, the lower sampling frequency cannot describe the wave information well. On the other hand, the higher sampling frequency will produce micronoise that influenced the results. Therefore, this paper, based on the research of previous scholars (such as Andersen et al. [4, 31] and Huang and Tauchen [32], Huang et al. [33]) and the influence of both hands, uses the CSI 300 index of 5 minutes high frequency data. After eliminating the trading time related data and supplementing the missing data using moving average interpolation method, there are 58751 data, that is, 49 data everyday. (including 1 overnight trading data and 48 intraday trading data). In this paper, we need to use the variables; the rate of return $R_{t}$, the logarithmic realized volatility $\ln \left(\mathrm{RV}_{t}\right)$, the logarithmic continuous sample path variation $\ln \left(C_{t}\right)$, and the logarithmic discontinuous jump variation $\ln \left(J_{t}+1\right)$ are all obtained by Matlab 2013a or Excel 2007.

Table 1 is descriptive statistics results of $R_{t}, \ln \left(R V_{t}\right)$, $\ln \left(C_{t}\right)$, and $\ln \left(J_{t}+1\right)$. From Table 1 , we can find that $\ln \left(\mathrm{RV}_{t}\right)$ sequence shows the phenomenon of "High Kurtosis and Fat Tail" and does not obey the normal distribution; this shows that China's stock market volatility is large. In addition, the unit root test (ADF test) shows that every sequence in the $99 \%$ confidence interval significantly declined to unit root of null hypothesis, so each sequence is stationary, and we can further analyze the models.

4.2. Parameter Estimation. In Section 2, we introduce Bayesian estimation results of the SV, SV-RV, and SV-CJ model using the MCMC methods through the OpenBUGS software. In order to ensure the convergence of the estimated parameters, 50000 iterations are performed on each parameter in the process of Gibbs sampling; by observing the orbit of the parameters iterations and the autocorrelation function, we found that after 10,000 iterations, the iterative process has converged. Hence, we anneal by using the first
10,000 samples and estimate the model by using the last 40,000 samples in this paper.

Table 2 lists the results of the Bayesian parameter estimation of the SV, SV-RV, and SV-CJ model, including the mean, standard deviation, MC error (the error of Monte Carlo simulation value), 95\% confidence interval for the posterior, the median, and the value of the deviation information criterion (DIC) of the parameter estimation. Firstly, we analyze the results of Bayesian estimation of the SV and SV-RV model. The estimation value of the parameter $\beta$ is close to 1, explaining that there is a strong persistence and autocorrelation with the return volatility of China's stock market, in accord with mature capital markets (such as the US and UK) and emerging capital markets (such as South Korea and New Zealand). If the standard deviation and $\mathrm{MC}$ error of the parameter are small, the accuracy of the parameter estimation is much higher. In the SV-RV model, estimation of parameter $\gamma$ is positive; its standard deviation and MC errors are relatively small and 95\% confidence interval for the posterior does not contain the value 0 , which proves that the prior logarithmic realized volatility $\ln \left(\mathrm{RV}_{t-1}\right)$ has a significant impact on the current volatility. $\ln \left(\mathrm{RV}_{t-1}\right)$ contains certain volatility forecast information. Comparing the DIC of SV model and SV-RV model, we find that the DIC of SV-RV model is smaller than that of SV model, which shows that the SV-RV model has a better measuring accuracy to the volatility and agrees with the research results of Koopman et al. [23] and Jacquier and Miller [26].

We focus on the estimation results of SV-CJ model; the coefficient $\lambda$ of the logarithmic continuous sample path variation $\ln \left(C_{t-1}\right)$ in the model is positive; its standard deviation and MC errors are relatively small and 95\% confidence interval for the posterior does not contain the value 0 , which proves that the prior logarithmic continuous sample path variation $\ln \left(C_{t-1}\right)$ has a certain prediction on the current volatility. However, the coefficient $\theta$ of the logarithmic discontinuous jump variation $\ln \left(J_{t-1}+1\right)$ in the model is comparatively large and $95 \%$ confidence interval for the posterior contains the value 0 , proving that the previous logarithmic discontinuous jump variation $\ln \left(J_{t-1}+1\right)$ has little effect on the current volatility. In addition, comparing the DIC of the models, we can find that the DIC of SV-CJ model is smaller than that of the SV-RV model, which shows that the SV-CJ model has a better measuring accuracy to the volatility and shows that adding the decomposition of the logarithmic realized volatility to the volatility equation SV model can improve the measurement capability to the return volatility. Therefore, when measuring the return volatility, it is more reasonable to use the SV-CJ, SV and SV-RV model.

\subsection{Forecasting}

4.3.1. In-Sample Forecasts. Figure 1 contains a real volatility sequence and three in-sample forecast volatility sequences that are obtained by the SV, SV-RV, and SV-CJ model. To comparatively analyze the predictive accuracy for future volatility of the SV, SV-RV, and SV-CJ model, we use the loss functions and SPA test to compare the predictive accuracy of 
TABle 1: Descriptive statistics for each variable.

\begin{tabular}{lcccccc}
\hline & Mean & Std. dev. & Skewness & Kurtosis & Jarque-Bera & ADF-t statistic \\
\hline$R_{t}$ & -0.0152 & 2.1141 & -0.2647 & 4.9345 & $200.95^{* * *}$ & $-32.901^{* * *}$ \\
$\ln \left(\mathrm{RV}_{t}\right)$ & 0.9600 & 0.9323 & 0.5180 & 3.2163 & $55.961^{* * *}$ & $-4.9183^{* * *}$ \\
$\ln \left(C_{t}\right)$ & 0.7650 & 0.8992 & 0.3525 & 2.8382 & $26.138^{* * *}$ & $-5.3556^{* * *}$ \\
$\ln \left(J_{t}+1\right)$ & 0.2825 & 0.6209 & 3.0445 & 14.085 & $7990.6^{* * *}$ & $-16.266^{* * *}$ \\
\hline ****
\end{tabular}

${ }^{* * *}$ Indicates significance at the $1 \%$ level.

TABLE 2: The estimation results of the SV, SV-RV, and SV-CJ model.

\begin{tabular}{lcccccccc}
\hline Model & Parameter & Mean & Standard deviation & MC error & $2.5 \%$ quantile & Median & 97.5\% quantile & DIC \\
\hline \multirow{2}{*}{ SV } & $\alpha$ & 0.0377 & 0.0178 & 0.0011 & 0.0136 & 0.0347 & 0.0798 & 4961.0 \\
& $\beta$ & 0.9694 & 0.0141 & 0.0009 & 0.9356 & 0.9860 & 0.9894 & 0.3381 \\
\multirow{3}{*}{ SV-RV } & $\alpha$ & 0.2053 & 0.0594 & 0.0037 & 0.1085 & 0.1982 & 0.7541 & 4951.0 \\
& $\gamma$ & 0.6063 & 0.0886 & 0.0057 & 0.4152 & 0.6175 & 0.4132 \\
\hline \multirow{3}{*}{ SV-CJ } & $\alpha$ & 0.2870 & 0.0587 & 0.0037 & 0.1855 & 0.2823 & 0.4581 \\
& $\lambda$ & 0.2927 & 0.06966 & 0.0046 & 0.1665 & 0.2865 & 0.7192 \\
& $\lambda$ & 0.3566 & 0.05807 & 0.0038 & 0.2208 & 0.3623 & 0.4602 & 4938.0 \\
& $\theta$ & 0.0389 & 0.04991 & 0.0030 & -0.06129 & 0.03955 & 0.1409 \\
\hline
\end{tabular}

these three models in this paper. Table 3 lists the statistical results of the loss functions (the MAE, HMAE, MSE, HMSE, QLIKE, and $R^{2} L O G$ ) about the SV, SV-RV, and SV-CJ model in the in-sample forecasts. Table 4 lists the SPA test results of SV, SV-RV, and SV-CJ model in the sample in the in-sample forecasts. In Table 3, comparing the size of the loss functions, we find that apart from one point that the QLIKE of SV-RV model is slightly smaller than that of SV-CJ model, the other loss functions of SV model are greater than those of SV-RV model, and the loss functions of SV-RV model are greater than the loss functions of SV-CJ model. In Table 4, the first column represents the baseline model $M_{0}$. Numerical values in the table are the $P$ value of SPA test; the larger the $P$ value, the stronger the predictive accuracy of the baseline model $M_{0}$, compared with the other two comparison models. In this table, there are four $P$ values of SPA test treating the SV-CJ model as a baseline model larger than those of SV-RV model. Similarly, there are four $P$ values of SPA test treating SV-RV model as a baseline model larger than those of SV model.

Analyzing the results of the loss functions in Table 3 and the results of SPA test in Table 4, we can get the following conclusions. The in-sample forecast accuracy of SV model for return volatility is weaker than that of SV-RV model, and the in-sample forecast ability of SV or SV-RV model for return volatility is weaker than that of SV-CJ model.

4.3.2. Out-of-Sample Forecasts. Compared with the insample predictive accuracy of the model, we are more concerned with the out-of-sample forecasting accuracy, because the out-of-sample forecasting is more meaningful to financial practical issues like the financial risk management, financial asset pricing, financial derivatives pricing, and so on. In order to effectively evaluate the out-of-sample forecasting accuracy

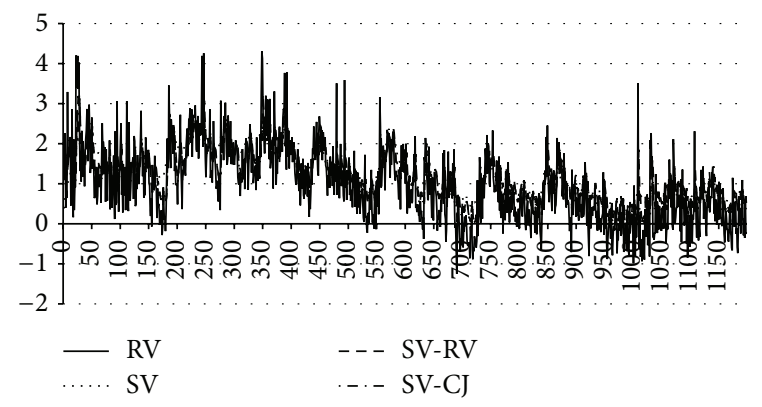

FIGURE 1: Comparison of the in-sample forecasting performance of the SV, SV-RV, and SV-CJ model. In the figure, RV represents the true volatility; SV, SV-RV, and SV-CJ represent the forecast volatility of the SV, SV-RV, and SV-CJ model, respectively.

of the models, we use the rolling time windows method to test the volatility forecasting accuracy of the SV, SV-RV, and SVCJ model. We select 999 samples as the fixed time windows and the last 200 samples (May 31, 2011-April 20, 2012) as the prediction and evaluation interval. Figure 2 contains a real volatility sequence and three out-of-sample forecast volatility sequences that are obtained by the SV, SV-RV, and SV-CJ model. The analysis approach is consistent with the in-sample forecasting part, still using the loss functions and SPA test to compare the predicting accuracy of each model. The results are shown in Tables 5 and 6 . In Table 5, comparing the loss functions, in these three models, apart from one point that the MSE of SV-CJ model is slightly larger than that of SV Model, the other loss functions of SV-CJ are smaller than those of SV and SV-RV model, and the loss functions of SV-RV model are greater than those of SV-CJ model. In addition, comparing the loss functions about the SV and SV-RV models, the loss 
TABLE 3: The loss functions of in-sample forecasts.

\begin{tabular}{lcccccc}
\hline & MAE & HMAE & MSE & HMSE & QLIKE & $R^{2}$ LOG \\
\hline SV & 2.3879 & 0.7237 & 1.0140 & 34.830 & 2.2034 & 0.4572 \\
SV-RV & 2.3197 & 0.7104 & 0.9862 & 32.099 & $\mathbf{2 . 1 9 4 1}$ & 0.4377 \\
SV-CJ & $\mathbf{2 . 2 9 5 1}$ & $\mathbf{0 . 6 7 3 6}$ & $\mathbf{0 . 8 4 4 0}$ & $\mathbf{3 1 . 1 6 7}$ & 2.1983 \\
\hline
\end{tabular}

The bold part is the minimum value of each loss function.

TABLE 4: The SPA test results of in-sample forecasts.

\begin{tabular}{lcccccc}
\hline & MAE & HMAE & MSE & HMSE & QLIKE & $R^{2}$ LOG \\
\hline SV & 0.0780 & 0.2056 & 0.1330 & 0.0538 & 0.1882 & 0.7944 \\
SV-RV & 0.0408 & $\mathbf{0 . 8 5 6 2}$ & 0.1382 & 0.0648 & 0.3784 & 0.5798 \\
SV-CJ & $\mathbf{0 . 5 8 1 6}$ & 0.5456 & $\mathbf{0 . 6 7 3 0}$ & $\mathbf{0 . 6 4 1 2}$ & $\mathbf{0 . 7 2 2 0}$ \\
\hline
\end{tabular}

Numerical values in the table represent $P$ value of SPA test obtained by 5000 Bootstrap simulation; the larger the $P$ value, the stronger the in-sample predictive accuracy of the baseline model, compared with the other two comparison models. The bold part is the maximum $P$ value of each model's SPA test.

TABLE 5: The loss functions of out-of-sample forecasts.

\begin{tabular}{lcccccc}
\hline & MAE & HMAE & MSE & HMSE & QLIKE & $R^{2}$ LOG \\
\hline SV & 1.3145 & 1.9953 & 1.0463 & $\mathbf{7 . 0 8 0 3}$ & 1.4152 & 0.6488 \\
SV-RV & 1.4186 & 3.1494 & 1.1515 & 8.2392 & 1.4127 & 0.7103 \\
SV-CJ & $\mathbf{1 . 2 6 3 6}$ & $\mathbf{1 . 4 3 5 4}$ & $\mathbf{0 . 8 9 3 2}$ & 7.6884 & $\mathbf{1 . 3 8 7 8}$ \\
\hline
\end{tabular}

The bold part is the minimum value of each loss function.

TABLE 6: The SPA test results of out-of-sample forecasts.

\begin{tabular}{lcccccc}
\hline & MAE & HMAE & MSE & HMSE & QLIKE & $R^{2}$ LOG \\
\hline SV & 0.2324 & 0.0300 & 0.0256 & $\mathbf{0 . 8 5 5 8}$ & 0.0616 & 0.1048 \\
SV-RV & 0.0262 & 0.0106 & 0.0248 & 0.0386 & 0.2538 & 0.0092 \\
SV-CJ & $\mathbf{0 . 7 7 2 0}$ & $\mathbf{0 . 6 6 1 6}$ & $\mathbf{0 . 7 9 7 0}$ & 0.0208 & $\mathbf{0 . 8 8 6 2}$ \\
\hline
\end{tabular}

Numerical values in the table represent the $P$ values of SPA test obtained by 5000 Bootstrap simulation; the larger the $P$ value, the stronger out-of-sample predictive accuracy of the baseline model, compared with the other two comparison models. The bold part is the maximum $P$ value of each model's SPA test.

functions of SV model are smaller than those of the SV-RV model apart from the HMSE value. In Table 6, there are five $P$ values of SPA test treating SV-CJ model as a baseline model larger than those of SV-RV model and five $P$ values of SPA test treating SV model as a baseline model larger than those of SV-RV model.

Analyzing the results of loss functions in Table 5 and the results of SPA test in Table 6, we can get the following conclusions. The out-of-sample forecasting accuracy of SVCJ model for return volatility is stronger than that of SV or SV-RV model. The out-of-sample forecast ability of SV model return volatility is stronger than that of SV-RV model.

All in all, by analyzing Sections 4.3.1 and 4.3.2, we know that the predictive accuracy for future volatility of SV-CJ model is the strongest in the above three volatility models. Therefore, adding the logarithmic realized volatility $\ln \left(\mathrm{RV}_{t-1}\right)$ to the $\mathrm{SV}$ volatility model and decomposing $\ln \left(\mathrm{RV}_{t-1}\right)$ into the logarithmic continuous sample path variation $\ln \left(C_{t-1}\right)$ and the logarithmic discontinuous jump variation $\ln \left(J_{t-1}+1\right)$, we can improve the model's performance to predict future volatility. Therefore, this decomposition is meaningful.

\section{Conclusion}

In this paper, we first construct the SV-CJ model based on SV-RV model. Then, we estimate the parameters of SV, SV$\mathrm{RV}$, and SV-CJ models through MCMC methods, using the 5 minutes frequency data in CSI 300 Index of China's stock market. Finally, using the loss functions and SPA test analyzes the return volatility forecasting accuracy of each model both in-sample and out-of-sample.

According to the parameter estimation results of the models, we find that the measuring accuracy for Chinese stock market volatility of SV-CJ model is significantly stronger than that of SV or SV-RV model. The prior logarithmic realized volatility and the prior logarithmic continuous sample path variation contain much predictive information on future volatility while the logarithmic discontinuous jump variation contains little predictive information. Moreover, comparative analysis of the predictive accuracy about the three models indicates that the in-sample forecasting accuracy for return volatility of SV-RV model is stronger than that of SV model. This conclusion may be different from the results of Koopman et al. [23], Jacquier and Miller [26]. It 


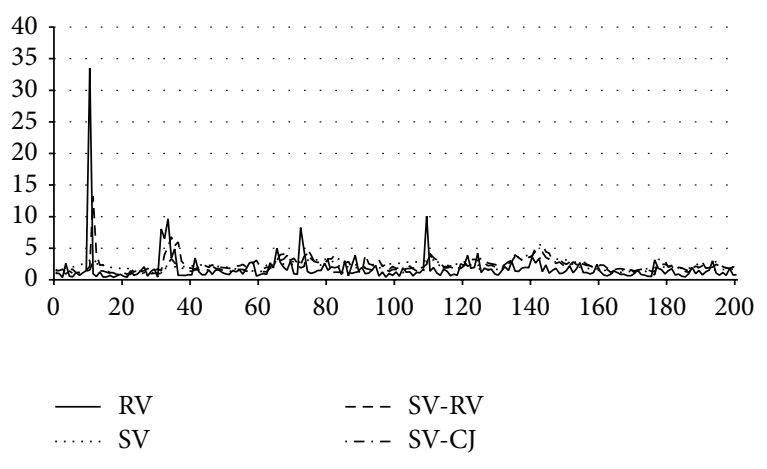

FIGURE 2: Comparison of the out-of-sample forecasting performance of the SV, SV-RV, and SV-CJ model. In the figure, RV represents the true volatility; SV, SV-RV, and SV-CJ represent the forecast volatility of the SV, SV-RV, and SV-CJ model, respectively.

may be due to the inconsistency with the model predictive accuracy of future volatility in different markets. In the Chinese stock market, the performance of SV-RV model added RV as the exogenous variables to predict the stock volatility are not significantly stronger than the SV model. However, the volatility forecasting accuracy of SV-CJ model is significantly stronger than the other two models, which shows that using SV-CJ model to measure and predict the volatility is more reasonable in financial practical issues like the financial risk management, financial asset pricing, and financial derivatives pricing. While the SV-CJ model has a better accuracy on volatility measuring and forecasting, it is still necessary to improve the measuring precision and forecasting precision of the volatility model. Therefore, we will further focus on the study to improve the measuring accuracy and forecasting accuracy of the volatility models on the basis of SV-CJ model.

\section{Conflict of Interests}

The authors declare that there is no conflict of interests regarding the publication of this paper.

\section{Acknowledgment}

This work was supported in part by the Natural Science Foundation of China (no. 71171024, no. 71371195, and no. 71221061).

\section{References}

[1] F. Corsi, "A simple approximate long-memory model of realized volatility," Journal of Financial Econometrics, vol. 7, no. 2, pp. 174-196, 2009.

[2] F. Wen and X. Yang, "Skewness of return distribution and coefficient of risk premium," Journal of Systems Science \& Complexity, vol. 22, no. 3, pp. 360-371, 2009.

[3] J. Liu, C. Ma, and F. Wen, "An Actuarial approach to option pricing under O-U process and stochastic interest rates," in Proceedings of the International Joint Conference on Computational
Sciences and Optimization (CSO '09), pp. 549-553, IEEE, Sanya, China, April 2009.

[4] T. G. Andersen, T. Bollerslev, and X. Huang, "A reduced form framework for modeling volatility of speculative prices based on realized variation measures," Journal of Econometrics, vol. 160, no. 1, pp. 176-189, 2011.

[5] T. G. Andersen, D. Dobrev, and E. Schaumburg, "Jump-robust volatility estimation using nearest neighbor truncation," Journal of Econometrics, vol. 169, no. 1, pp. 75-93, 2012.

[6] Z. Dai, D. Li, and F. Wen, "Robust conditional value-atrisk optimization for asymmetrically distributed asset returns," Pacific Journal of Optimization, vol. 8, no. 3, pp. 429-445, 2012.

[7] F. Wen, Z. Li, C. Xie, and D. Shaw, "Study on the fractal and chaotic features of the Shanghai composite index," Fractals, vol. 20, no. 2, pp. 133-140, 2012.

[8] T. Bollerslev, D. Osterrieder, N. Sizova, and G. Tauchen, "Risk and return: long-run relations, fractional cointegration, and return predictability," Journal of Financial Economics, vol. 108, no. 2, pp. 409-424, 2013.

[9] T. Bollerslev, V. Todorov, and S. Z. Li, "Jump tails, extreme dependencies, and the distribution of stock returns," Journal of Econometrics, vol. 172, no. 2, pp. 307-324, 2013.

[10] J. Liu, L. Yan, and C. Ma, "Pricing options and convertible bonds based on an actuarial approach," Mathematical Problems in Engineering, vol. 2013, Article ID 676148, 9 pages, 2013.

[11] J. Liu, M. Tao, C. Ma, and F. Wen, "Utility indifference pricing of convertible bonds," International Journal of Information Technology \& Decision Making, vol. 13, no. 2, pp. 429-444, 2014.

[12] J. Liu, J. Xiao, L. Yan, and F. Wen, "Valuing catastrophe bonds involving credit risks," Mathematical Problems in Engineering, vol. 2014, Article ID 563086, 6 pages, 2014.

[13] R. F. Engle, "Autoregressive conditional heteroscedasticity with estimates of the variance of United Kingdom inflation," Econometrica, vol. 50, no. 4, pp. 987-1007, 1982.

[14] T. Bollerslev, "Generalized autoregressive conditional heteroskedasticity," Journal of Econometrics, vol. 31, no. 3, pp. 307327,1986

[15] J. S. Taylor, Modeling Financial Time Series, John Wiley \& Sons, Chichester, UK, 1986.

[16] J. Danielsson, "Stochastic volatility in asset prices estimation with simulated maximum likelihood," Journal of Econometrics, vol. 64, no. 1-2, pp. 375-400, 1994.

[17] F. C. Wang, L. X. Jiang, and G. Li, "Estimating volatility of Chinese stock market by stochastic volatility model," Journal of Management Sciences in China, vol. 4, pp. 63-72, 2003.

[18] S. Kim, N. Shephard, and S. Chib, "Stochastic volatility: likelihood inference and comparison with ARCH models," Review of Economic Studies, vol. 65, no. 3, pp. 361-393, 1998.

[19] J. Yu, "Forecasting volatility in the New Zealand stock market," Applied Financial Economics, vol. 12, no. 3, pp. 193-202, 2002.

[20] P. Sadorsky, "A comparison of some alternative volatility forecasting models for risk management," in Proceedings of the 2nd IASTED International Conference: Financial Engineering and Applications, 2004.

[21] C. Pederzoli, "Stochastic volatility and GARCH: a comparison based on UK stock data," The European Journal of Finance, vol. 12, no. 1, pp. 41-59, 2006.

[22] Y. Wei, "Forecasting volatility of fuel oil futures in China: GARCH-type, SV or realized volatility models?" Physica A: Statistical Mechanics and its Applications, vol. 391, no. 22, pp. 5546-5556, 2012. 
[23] S. J. Koopman, B. Jungbacker, and E. Hol, "Forecasting daily variability of the S\&P 100 stock index using historical, realised and implied volatility measurements," Journal of Empirical Finance, vol. 12, no. 3, pp. 445-475, 2005.

[24] T. G. Andersen and T. Bollerslev, "Answering the critics: yes, ARCH models do provide good volatility forecasts," International Economic Review, vol. 4, pp. 885-905, 1998.

[25] J. Geweke, G. Koop, and H. Dijk, The Oxford Handbook of Bayesian Econometrics, Oxford University Press, 2011.

[26] E. Jacquier and S. Miller, "The information content of realized volatility," Working Paper, HEC Montreal, 2013.

[27] O. E. Barndorff-Nielsen and N. Shephard, "Power and bipower variation with stochastic volatility and jumps," Journal of Financial Econometrics, vol. 2, pp. 1-37, 2004.

[28] O. E. Barndorff-Nielsen and N. Shephard, "Econometrics of testing for jumps in financial economics using bipower variation," Journal of Financial Econometrics, vol. 4, pp. 1-30, 2006.

[29] P. R. Hansen, "A test for superior predictive ability," Journal of Business \& Economic Statistics, vol. 23, no. 4, pp. 365-380, 2005.

[30] M. Martens, "Measuring and forecasting S\&P 500 indexfutures volatility using high-frequency data," Journal of Futures Markets, vol. 22, no. 6, pp. 497-518, 2002.

[31] T. G. Andersen, T. Bollerslev, and F. X. Diebold, "Roughing it up: including jump components in the measurement, modeling, and forecasting of return volatility," The Review of Economics and Statistics, vol. 89, no. 4, pp. 701-720, 2007.

[32] X. Huang and G. Tauchen, "The relative contribution of jumps to total price variance," Journal of Financial Econometrics, vol. 3 , no. 4, pp. 456-499, 2005.

[33] C. Huang, X. Gong, X. Chen, and F. Wen, "Measuring and forecasting volatility in Chinese stock market using HAR-CJM model," Abstract and Applied Analysis, vol. 2013, Article ID 143194, 13 pages, 2013.

[34] E. Ruiz, "Quasi-maximum likelihood estimation of stochastic volatility models," Journal of Econometrics, vol. 63, no. 1, pp. 289-306, 1994.

[35] E. Jacquier, N. G. Polson, and P. E. Rossi, "Bayesian analysis of stochastic volatility models," Journal of Business and Economic Statistics, vol. 12, no. 4, pp. 371-417, 1994.

[36] T. G. Andersen and B. E. Sørensen, "GMM estimation of a stochastic volatility model: a Monte Carlo study," Journal of Business and Economic Statistics, vol. 14, no. 3, pp. 328-352, 1996.

[37] T. Watanabe, "A non-linear filtering approach to stochastic volatility models with an application to daily stock returns," Journal of Applied Econometrics, vol. 14, no. 2, pp. 101-121, 1999.

[38] J. Durbin and S. J. Koopman, Time Series Analysis by State Space Methods, vol. 38, Oxford University Press, Oxford, UK, 2nd edition, 2012.

[39] L. Bauwens and M. Lubrano, "Bayesian inference on GARCH models using Gibbs sampler," Econometrics Journal, vol. 1, pp. 23-46, 1998.

[40] D. J. Spiegelhalter, N. G. Best, B. P. Carlin, and A. van der Linde, "Bayesian measures of model complexity and fit," Journal of the Royal Statistical Society B, vol. 64, no. 4, pp. 583-639, 2002.

[41] A. P. Dempster, "The direct use of likelihood for significance testing," in Proceedings of Conference on Foundational Questions in Statistical Inference, pp. 335-352, Department of Theoretical Statistics, University of Aarhus, 1974.

[42] T. Bollerslev, R. F. Engle, and D. Nelson, "ARCH models," in Handbook of Econometrics, D. L. McFadden, Ed., vol. 4, pp.
2961-3038, Elsevier Science B.V., Amsterdam, The Netherlands, 1994.

[43] P. R. Hansen and A. Lunde, "A forecast comparison of volatility models: does anything beat a GARCH $(1,1)$ ?" Journal of Applied Econometrics, vol. 20, no. 7, pp. 873-889, 2005.

[44] G. M. Martin, A. Reidy, and J. Wright, "Does the option market produce superior forecasts of noise-corrected volatility measures?" Journal of Applied Econometrics, vol. 24, no. 1, pp. 77-104, 2009.

[45] Y. Wang and C. Wu, "Forecasting energy market volatility using GARCH models: can multivariate models beat univariate models?" Energy Economics, vol. 34, no. 6, pp. 2167-2181, 2012.

[46] J. C. Hung, T. W. Lou, Y. H. Wang, and J. Lee, "Evaluating and improving GARCH-based volatility forecasts with range-based estimators," Applied Economics, vol. 45, no. 28, pp. 4041-4049, 2013.

[47] H. White, "A reality check for data snooping," Econometrica, vol. 68, no. 5, pp. 1097-1126, 2000. 


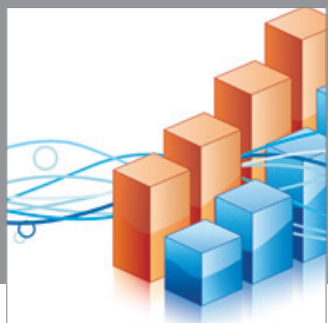

Advances in

Operations Research

mansans

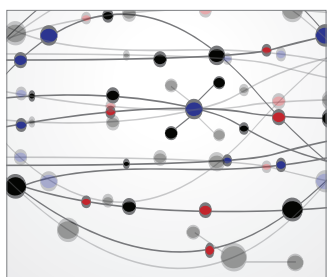

The Scientific World Journal
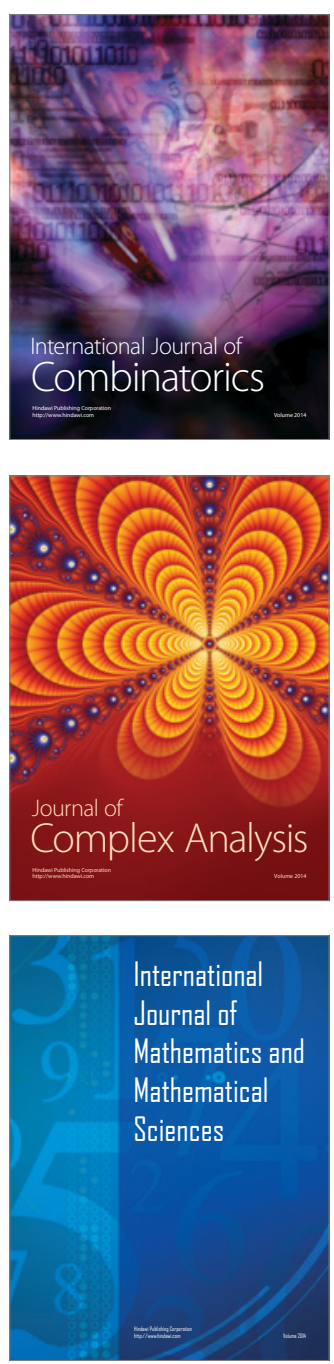
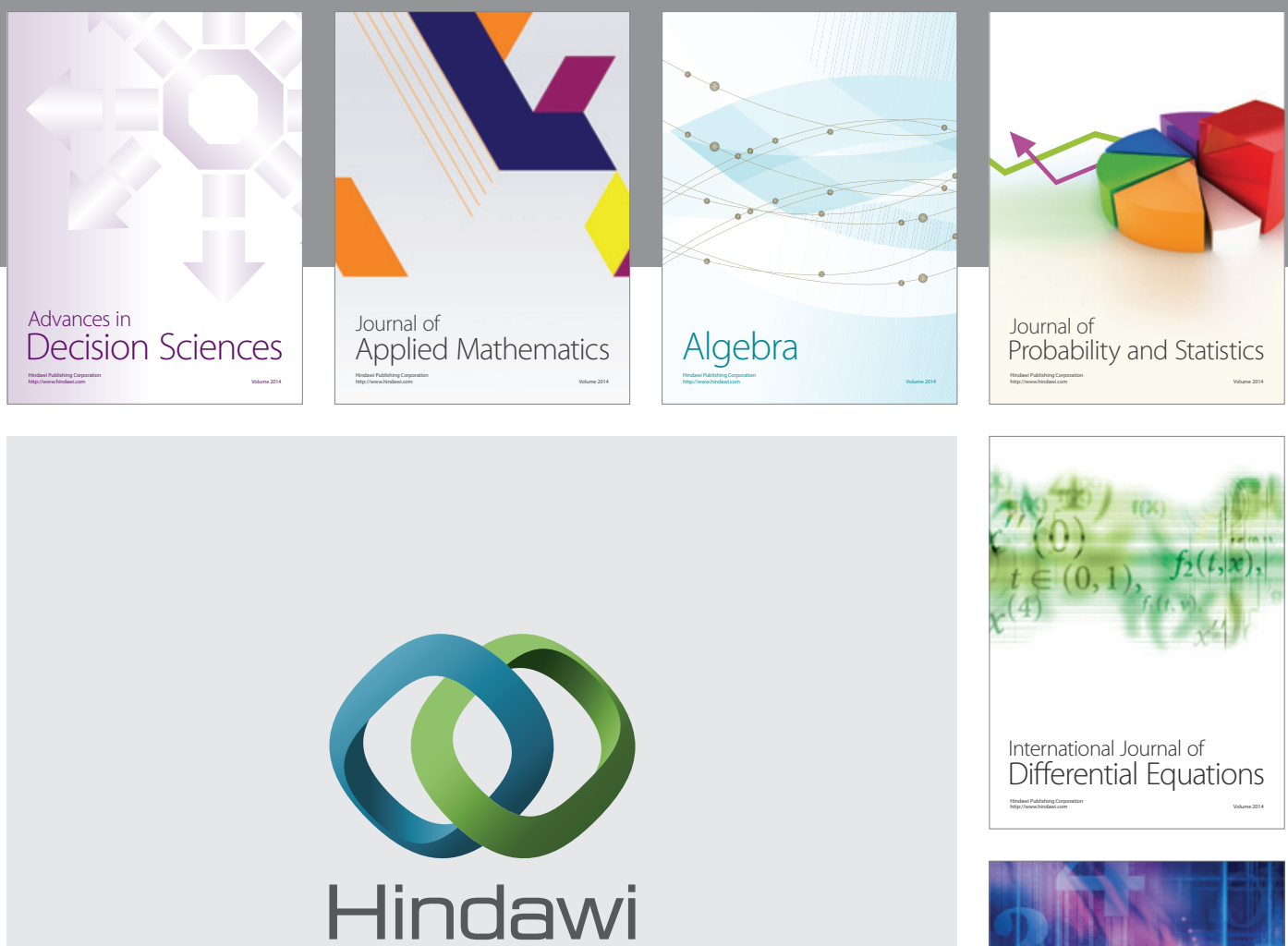

Submit your manuscripts at http://www.hindawi.com
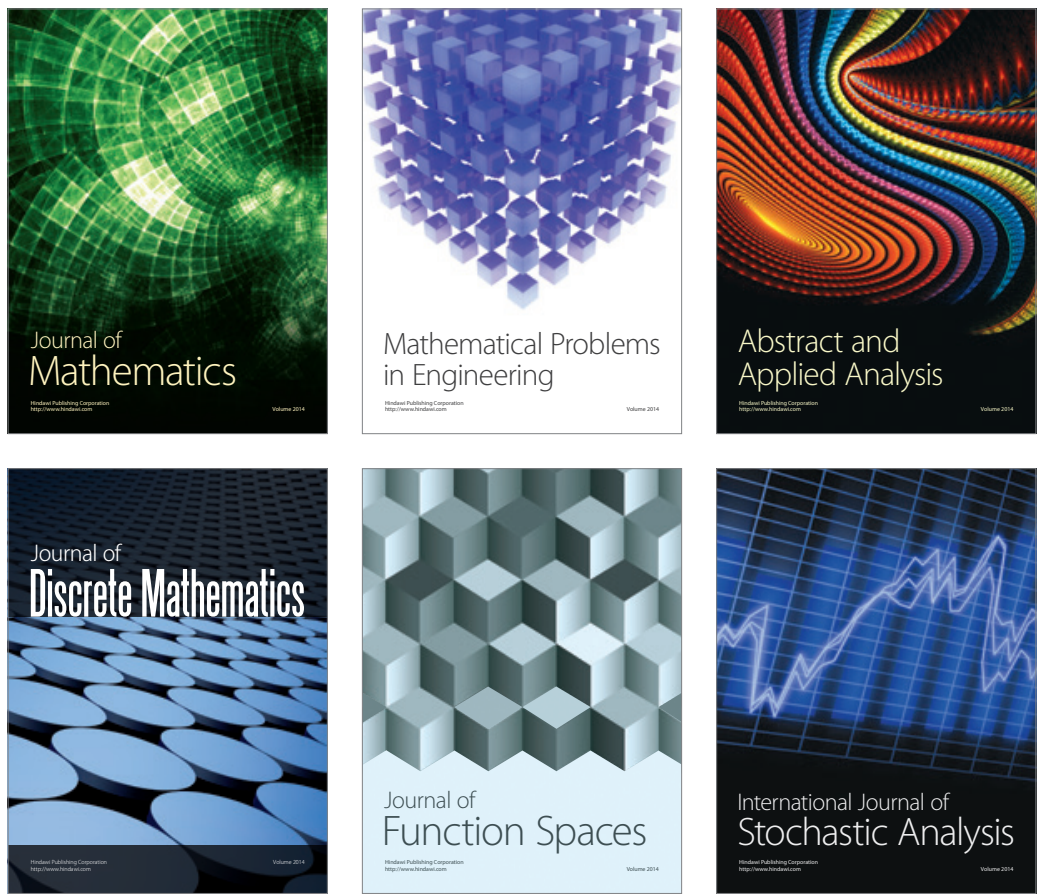

Journal of

Function Spaces

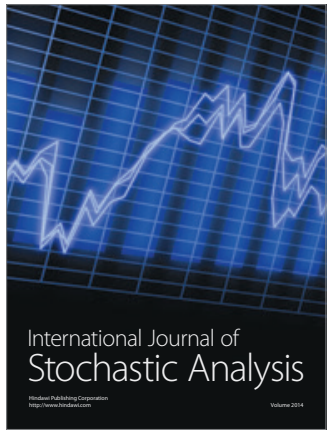

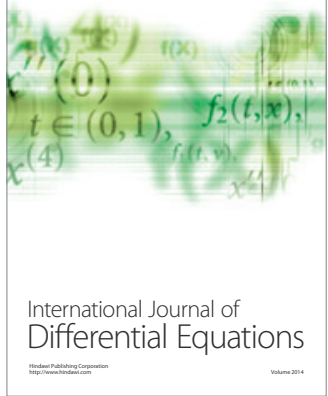
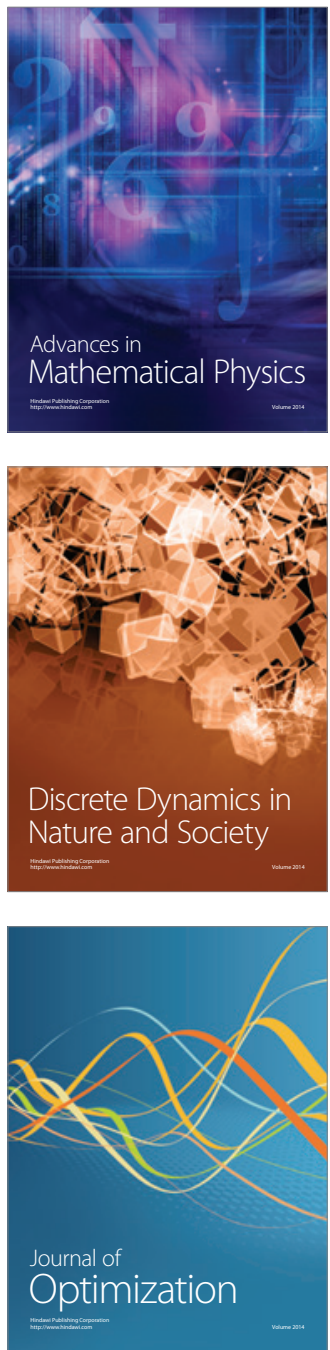